\title{
Hybrid Precoding Design Using MMSE Baseband Precoder for mm-Wave Multi-User MIMO Systems
}

\author{
Farah Raisa, Md. Rafiqul Islam, Khaizuran Abdullah, Asif Reza
}

\begin{abstract}
For future $5 G$ wireless communication networks, millimeter-wave (mmWave) cellular systems is considered to be the key enabling technology because of its high data rates, low latency, high system capacity, and huge available bandwidths. However, multiuser networks in mm Wave frequency bands encounter high path loss and interference, thus degrading the performance. Applying large antenna arrays at the base stations $(B S)$ in order to achieve high beamforming gains with the help of precoding techniques is an efficient way of improving the performance of the system. Although multi-user beamforming can improve spectral efficiencies, full digital beamforming strategies used in the conventional microwave systems increase the hardware cost and consumes high power for large number of antennas in mmW systems. In this paper, a low-complexity multi-user hybrid precoding structure is proposed for mm Wave multiple input multiple output (MIMO) channels utilizing Minimum Mean Square Error (MMSE) precoders at the BS with perfect channel knowledge. Simulations show that the achievable rate obtained by the proposed hybrid precoding scheme is very close to the single-user rate and also performs better compared to other hybrid precoding approaches.
\end{abstract}

Keywords: MIMO, Millimeter-wave, mmWave, Hybrid Precoding, MMSE

\section{INTRODUCTION}

To obtain high performance and throughput, millimeter wave networks are one of the efficient technolo-gies which can be utilized to apply in future $5 \mathrm{G}$ wireless systems. The spectrum band that ranges from $30 \mathrm{GHz}$ to $300 \mathrm{GHz}$ is generally known as the mm-waves frequencies [1]. As millimeter-Wave (mmW) spectrum has very small wavelength, so the available bandwidths are larger than the bandwidth those are used today. As a result, there is more available spectrum and a large number of small antennas can be used in this frequency band which will lead to great-er system capacity. So, it is expected to play an important role for the future $5 \mathrm{G}$ wireless networks [2]. However, there are a number of challenges that mmWave is going to face and before making it widely used, it must be managed and solved in order to ensure maximum efficiency. Millimeter-Wave is

Revised Version Manuscript Received on 10 September, 2019.

Farah Raisa, Department of Electrical and Computer Engineering,

Md. Rafiqul Islam, Department of Electrical and Computer Engineering, International Islamic University Malaysia, Kuala Lumpur Malaysia

Khaizuran Abdullah, Department of Electrical and Computer Engineering, International Islamic University Malaysia, Kuala Lumpur Malaysia

Asif Reza, Department of Electrical and Computer Engineering, International Islamic University Malaysia, Kuala Lumpur Malaysia International Islamic University Malaysia, Kuala Lumpur Malaysia

subjected to severe attenuation and path loss due to noise and interference. So, these problems can be solved by antenna Beamforming and multi user MIMO.

Implementation of analog beamforming for mmWave systems using phase-shifters is popular due to low cost and simplicity of the structure [3-6]. The trans-ceivers operating at high frequencies as in the case of mil-limeter wavebands support analog beamforming since the digital beamforming makes the system more complex with a large number of antennas. Analog beamforming has been the major support particularly for the indoor mm-wave systems adopting different beamforming training protocols [3-4]. To overcome the high path loss issue of the mm-wave frequencies, a large number of antennas are aligned and the RF chains are kept as minimum as possible to maintain simple circuit and low power consumption. The beam pointing angles are needed to be aligned to face each other to form beam angle pair. However, these proposed methods were only applicable for single-stream transmission and also has large training overhead for large number of users. The performance of these low-power analog beamforming approaches, is very poor and not completely suitable for mm-wave channels. Fully digital beamforming architectures used in broadband microwave [7] is impractical for millimeter-wave systems due to high hardware complexity and power consumption.

The efficient beamforming architecture for mm-wave systems needs to be designed with both analog and digital processing [8-17]. Hybrid analog-digital beamform-ing involves dividing the MIMO processing into both ana-log and digital domain for multi-user data transmission with minimum interference. Limited number of radio frequency (RF) chains are utilized that forwards the processing of the digital baseband superseded by the analog beamforming. In order to maximize the average received SNR and thus the link budget, joint analog-digital beamforming algorithm was developed [9] with perfect Channel State Information (CSI) where the number of radio frequency chains were reduced with negligible performance loss to minimize the cost. Zero forcing $(\mathrm{ZF})$ and phased zero-forcing (PZF) hybrid precoding scheme is proposed in several literatures [14] [15] performing low-dimensional zero forcing precoding at the baseband. The analog RF precoder is designed to obtain the large array gain

and after that $\mathrm{ZF}$ precoding is carried out depending on the channel. 


\section{HYBRID PRECODING DESIGN USING MMSE BASEBAND PRECODER FOR MM-WAVE MULTI-USER MIMO SYSTEMS}

The hybrid BF algorithm for single-user mm-wave systems which assumes only limited knowledge of the channel [14] considers both the reciprocal and sparse envi-ronment. However, these proposed hybrid precoding algo-rithms do not utilize the characteristics of the mm-wave channel properly. As a result, when applied to $\mathrm{mmW}$ sys-tems, these approaches will consume high power due to the use of high resolution $\mathrm{ADC}$ at the receiver. Efficient beam-forming design for millimeter-wave channels considering the special characteristics of the channel is needed that can bring benefit in practical application for the massive num-ber of antennas and which is compatible for millimetre-wave frequency bands.

In this paper, an efficient hybrid precoding design for mmWave channel with comparatively low-complexity is proposed where precoding is done in both analog and digital domains for multi-user scenario. In this design, RF precoding is done to increase the transmit power and after that MMSE baseband precoding is adapted for multi-user system to mitigate the inter-user interference. Rest of the paper is organized as follows: The system model for the mmWave hybrid beamforming structure in discussed in section 2 . In section 3, the proposed hybrid MMSE precod-ing design is presented and analysed under perfect CSI in section 4 . Simulation results is illustrated in section 5 and finally the paper is concluded in section 6 .

\section{SYSTEM MODEL}

In the system model for mmWave propagation, it is considered that $N_{t}$ number of transmit antennas and $N_{R F}$ number of chains are connected at the BS. The base station communicates with the mobile stations of ${ }^{K}$ users, each of them equipped with $N_{r}$ number of receive antennas. In multi-user beamforming system, each of the users supports single-stream transmission between the BS and the MS. So, the number of data streams is equal to the number of total user $K$. It is also considered that the number of RF chains at the BS is same as the highest number of users being served at the same time i.e., the number of users are less than the number of $\mathrm{RF}$ chains. Moreover, it is assumed that ${ }^{K}$ number of $N_{R F}$ will be used by the base station in order to serve the users. The transmitter at the BS executes $K \times K_{\text {baseband precoder, }} \mathbf{F}_{B}$, and $N_{t} \times K$ RF precoder, $\mathbf{F}_{R}$. The transmitted signal is then given by,

$$
x=\mathrm{F}_{R} \mathrm{~F}_{B}
$$

In this equation, ${ }^{s}$ is the $K \times 1$ transmitted symbol vector such that, $\mathbb{E}\left[s s^{*}\right]=\frac{P}{K} I_{K}$, where, ${ }^{P}$ is the average transmit power. Analog phase shifters are being used to execute $\mathbf{F}_{R}$, so its entries are normalized in order to satisfy $\left\|\left[\mathrm{F}_{R}\right]_{\mathrm{m}, \mathrm{n}}\right\|^{-2}=N_{\mathrm{t}}^{-1}$. Moreover, each entry of $\mathbf{F}_{R}$ varies in phase-only and we normalize the magnitude so that it satisfies $\left[\mathrm{F}_{R}\right]_{\mathrm{m}_{n} n}=\frac{1}{\sqrt{\mathrm{N}_{L}}} e^{j \phi_{m, n}}$ total power constraints at the BS is accomplished by normalizing $\mathrm{F}_{B}$ such that $\left\|\mathrm{F}_{\mathrm{R}} \mathrm{F}_{B}\right\|^{2}=K$. channel model is adopted [14] [17] where received signal observed by the $k^{\text {th }}$ user,

$$
r_{k}=\mathbf{H}_{k} \sum_{n=1}^{K} \mathbf{F}_{R} f_{n}^{B} s_{n}+n_{k}
$$

Where, $\mathbf{H}_{k}$ is the mm-wave channel matrix between BS and MS with dimension $N_{r} \times N_{t}, n_{k}$ is the additive Gaussian noise and $n_{k} \sim \mathcal{N}\left(0, \sigma^{2} I\right)$.

\section{A. Channel Model}

The hybrid precoders $\left(\mathbf{F}_{R}, \mathbf{F}_{B}\right)$ and the hybrid combiners $\left(\mathbf{W}_{R}\right)$ are investigated depending on the channel matrix $\mathbf{H}_{\text {. In }}$ order to analysis the performance of the proposed hybrid precoding, a geometric mm-wave channel model is adopted with limited scattering characteristics for the channel, with each scatterer having a single propagation path. It is assumed that the channel is the sum of propagation paths, each having a scatterer and having $L_{K}$ number of scattering clusters for the channel ${ }^{k}$. Channel $\mathbf{H}_{k}$ from [14]can be expressed as

$$
\mathbf{H}_{k}=\sqrt{\frac{N_{t} N_{r}}{L_{k}}} \sum_{l=1}^{L_{k}} \alpha_{k, l} \mathrm{a}_{r}\left(\theta_{k_{z} l}\right) \mathrm{a}_{t}^{*}\left(\phi_{k_{j} l}\right)
$$

Where, ${ }^{\theta_{k_{\Perp}} \mathbb{1}}$ is the angle of arrival (AoA) and $\phi_{k_{\Perp} \rrbracket}$ is the angle of departure (AoD) of the $1^{\text {th }}$ path. ${ }^{\alpha_{k} \mathbb{l}}$ is the ${ }^{i^{i}}$ th path's complex gain adding the path loss, and $\mathrm{a}_{y}\left(\theta_{k_{\lambda} D}\right)$ and $\mathrm{a}_{\mathrm{t}}\left(\phi_{k_{2}}\right)$ are the array response vector of the antennas of the MS and $\mathrm{BS}$ respectively.

The simulation of the proposed design is based on two types of antenna arrays, i.e., uniform planar arrays (UPA) and uniform linear arrays (ULA). The array response vector for ULA antennas at the BS can be defined as

$$
\begin{aligned}
& \mathrm{a}_{\mathrm{t}}\left(\phi_{k_{i} D}\right)=\frac{1}{\sqrt{N_{\mathrm{t}}}}\left[1, \exp \left(j \frac{2 \pi}{\mathrm{a}} d \sin \left(\phi_{k_{i} \lambda}\right)\right), \ldots, \exp \left(j \left(N_{\mathrm{t}}-\right.\right.\right. \\
& \text { 1) } \left.\left.\frac{2 \pi}{\mathrm{a}} d \sin \left(\phi_{k_{i} I}\right)\right)\right]^{T}
\end{aligned}
$$

Where, is thecarrier wavelength, is the distance between two antenna elements.

\section{HYBRID MMSE PRECODING FOR ULTI-USER MIMO}

Designing hybrid precoders at the BS and combin-ers at the MSs efficiently to maximize the total sum rate of the system is the main objective of this thesis. The achieva-ble rate for the user at the MS can be written as in [14], after processing the received signal utilizing the combining vector,

$$
R_{k}=\log _{2}\left[1+\frac{\frac{F}{K}\left|w_{k}^{*} H_{k} F_{k}^{B}\right|^{2}}{\frac{P}{K} \sum_{n=k_{K}} \frac{P}{K}\left|w_{k}^{2} H_{k} f_{k}^{B}\right|^{2}+\sigma^{2}}\right]
$$

Total sum-rate of the system is given by,

$$
R=\sum_{k=1}^{K} R_{k}
$$

There are only certain values for the analog pre-coding and combining vectors because only the quantized angles are available for the analog phase-shifters.

There are only certain values for the analog precoding and combining vectors because only the quantized angles are available for the analog phase-shifters. These vectors are 
chosen from different beamforming codebooks. As the analog beamsteering codebooks are previously used and shown to have better performance for single-user precoding schemes, in this paper this beamstearing codebooks are adopted for selecting the RF beamforming vectors. Here, for channels with single path, the beamforming vectors have similar type of array response vector which can be represented by a simple angle. Let ${ }^{\mathcal{F}}$ and $\mathcal{W}$ be the RF precoding vector codebook and RF combining codebook respectively.

Considering the sum-rate as the performance metric, $\mathbf{F}_{R}^{*}$, $\left\{\mathrm{f}_{k}^{* B}\right\}_{k=1}^{K}$ and $\left\{w_{k}^{*}\right\}_{k=1}^{K}$ are needed to obtained from the equation (4) to solve

$$
\begin{aligned}
& \left.\left\{\mathrm{F}_{R^{*}}^{*}\left\{\mathrm{f}_{k}^{*}\right\}_{k=1}^{K}\right\}_{k}^{*}\left\{w_{k}^{*}\right\}_{k=1}^{K}\right\}=\arg \max \left(R_{g}\right) \\
& =\arg \max \sum_{k=1}^{K} \log _{2}\left(1+\frac{\frac{P}{K}\left|w_{k}^{*} H_{k} F_{R} \mathrm{f}_{k}^{B}\right|^{2}}{\frac{P}{K} \sum_{n \neq k}\left|w_{k}^{*} H_{k} F_{R} \mathrm{f}_{k}^{B}\right|^{2}+\sigma^{2}}\right)
\end{aligned}
$$

subject to, $\quad\left[\mathrm{F}_{R}\right]_{, k} \in \mathcal{F}_{s} k=1,2, \ldots, K$,

$\mathrm{w}_{\mathrm{k}} \in \mathcal{W}_{\mathrm{s}} k=1,2, \ldots, K_{v}$

$\left\|\mathbf{F}_{R}\left[\mathrm{f}_{1}^{B}, \mathrm{f}_{2}^{B}, \ldots, \mathrm{f}_{K}^{B}\right]\right\|_{F}^{2}=K$.

However, it is infeasible and unmanageable to obtain explicit solution of this maximization of the total sum-rate due to certain factors. The solution for the above equation needs to search for possible $\mathrm{F}_{R}$ and $\left\{w_{k}^{*}\right\}_{k=1}^{K}$ combinations over the $\mathcal{F}^{K} \times \mathcal{W}^{K}$. Moreover, the baseband precoder $\mathrm{F}_{B}$ is needed to incorporate with the RF beamforming vectors which needs the channel or effective channel feedback, resulting in large feedback and training overhead for this solution. A modified algorithm is proposed for mm-wave multi-user MIMO for a large number of antenna that can have better performance than the previous one with a very small training and feedback overhead.

The analog RF precoders and RF combiners are designed at the BS and MS respectively for single-user sce-nario avoiding the inter-user interference and the power of the signal per user is maximized without estimating the channel. The BF vectors for the kth user are designed in such a manner so that at the signal power is highest at the kth MS. The RF precoding scheme here is the typical ana-log precoding for single-user system that is used in other literatures [10] [12]. Efficient beam alignment algorithm is used which exploits the BF codebook to maximize the SNR. The hierarchical codebook utilizes a distance metric which can be optimized by using proper subarray methods.

After this stage, the digital baseband precoder is constructed for multi-user MIMO and inter-user interference in handled.

Effective

channels, $\bar{h}_{k}=\mathrm{w}_{k}^{*} \mathrm{H}_{k} \mathrm{~F}_{R}, k=1,2, \ldots, K_{x}$ are trained by the base station with the mobile stations. A random vector quantization (RVQ) codebook $(\mathcal{H})$ is used to quantize these effective channels by their corresponding $\mathrm{MS}(k)$, and then the index of the channel vector is fed to the base station with ${ }_{b}$ bits. The MMSE (Minimum Mean Squared Error) precoding is then constructed in the BS depending on quantized channels.

\section{PERFORMANCE ANALYSIS WITH PERFECT CSI}

In this section, the spectral efficiency is analysed which is achieved by the RF and baseband hybrid precoders. The performance is analysed for massive MIMO with a large number of transmit and receive antennas and with single-path channels. Assuming that the effective channel knowledge is perfect and the values of RF beamsteering angles are continuous. It is also assumed that all the codebooks including the RF codebooks for the for the BS and and MS and the RVQ codebook has the size of infinity. It is considered that the channel is single-path so the scattering path $L_{k}=1$ for $k=1,2,3 \ldots K$. The effective channel can be written for $k$ user after constructing the analog precoders, $\bar{h}_{k}=\mathrm{w}_{k} \mathrm{H}_{k} \mathrm{~F}_{k}=\sqrt{N_{t} N_{r}} a_{k} a_{t}^{*}\left(\phi_{k}\right) \mathrm{F}_{R}$

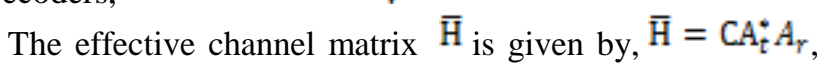
where ${ }^{C}$ is the diagonal matrix with dimension $K \times K$ and $[C]_{k_{i} k}=\sqrt{N_{t} N_{\gamma}} \alpha$. The MMSE precoder of digital baseband depending on the effective channels can be expressed as, $\mathrm{F}_{B=} \overline{\mathrm{H}}^{\mathrm{H}}\left(\overline{\mathrm{H}^{\mathrm{H}} \mathrm{H}+\alpha \mathrm{I}}\right)^{-1}$, where ${ }^{\alpha}$ is the normalization factor and it is normalized to adjust elements according to the power constraints $\left\|\mathrm{F}_{\mathrm{R}} \mathrm{f}_{\mathrm{k}}^{B}\right\|^{2}=1$. The elements of the diagonal

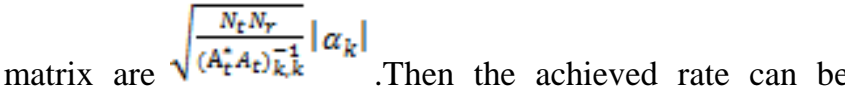
written as

$$
\begin{aligned}
& R_{k}=\log _{2}\left[1+\frac{S N R}{K}\left|\overline{\mathrm{h}}_{k}^{*} \mathrm{f}_{k}^{B}\right|^{2}\right]=\log _{2}[1+ \\
& \left.\frac{S N R}{K} \sqrt{\frac{N_{R} N_{r}}{\left.\left(A_{\mathrm{L}}^{*} A_{\mathrm{L}}\right)_{k}^{-1}\right]_{k}^{k}}}\left|\alpha_{k}\right|^{2}\right]
\end{aligned}
$$

For single-user, the achievable rate is given by

$$
R_{\text {single }}=\log _{2}\left[1+\frac{S N R}{K} N_{t} N_{r}\left|\alpha_{k}\right|^{2}\right]
$$

As the precoders and combiners are designed according to the algorithm considering the assumptions stated before, the achievable rate of user ${ }^{k}$ and the single user achievable rate increases with the similar slope when SNR is high. The difference between these two types of achievable rates is always constant as it only depends on the number of users and the number of transmitters.

$$
\text { The effective channel } \overline{\mathrm{H}}_{v} \text {, }
$$$$
\overline{\mathrm{H}}_{v}=\mathrm{D}_{v} P_{v}(10)
$$

$\mathrm{D}_{w}$ represents the diagonal matrix and $\mathrm{P}_{v}$ is the $K \times K$ matrix

Digital MMSE precoder,

$$
\mathrm{F}_{B}=\overline{\mathrm{H}}^{\mathrm{H}}\left(\overline{\mathrm{H}^{\mathrm{H}} \mathrm{H}}+\alpha \mathrm{I}\right)^{-1} \Lambda
$$

Where, $\alpha$ is the regularization factor and $\alpha=\frac{N_{r} \sigma_{m}^{2}}{\sigma_{n}^{2}}$, where $\sigma_{n}^{2}$ and $\sigma_{s}^{2}$ are noise variance and average transmit power respectively. So, the sum rate of user $K$ is given by 


$$
\mathrm{R}_{K}=\log _{2}\left(1+\frac{S N R}{K}\left|\bar{h}_{k}^{*} f_{k}^{B}\right|^{2}\right)
$$

This equation represents the achievable rate for user $\mathrm{k}$ obtained by the proposed hybrid precoding structure. In the next section, the results of the simulation comparing the

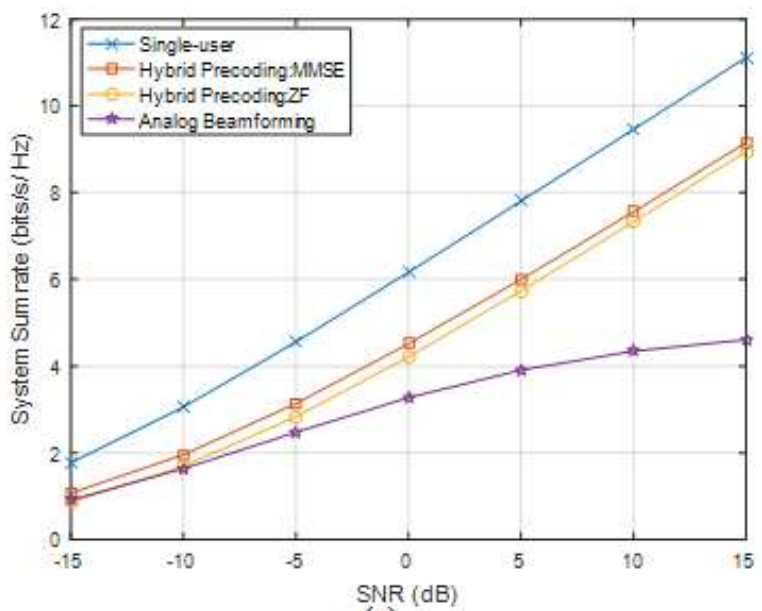

(a) spectral efficiencies of the proposed design and other different beamforming approaches are presented.

\section{V.SIMULATION RESULTS}

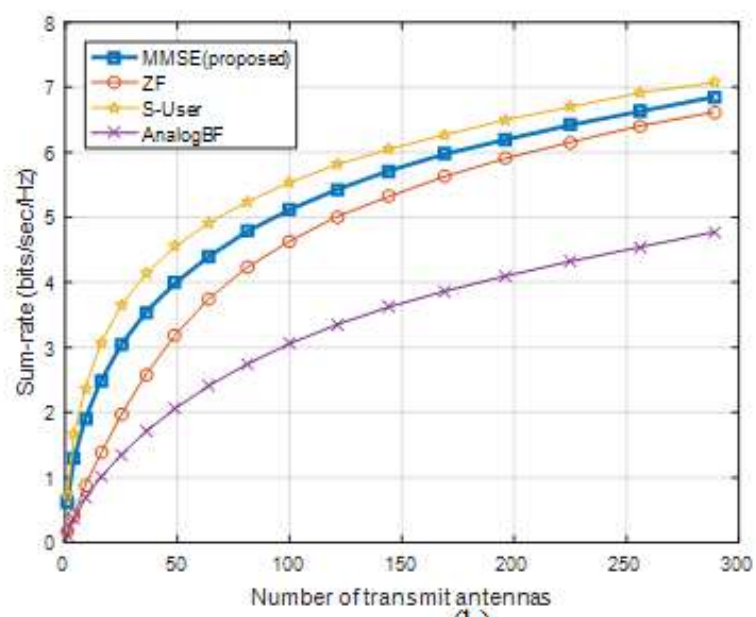

(b)

Fig. 1. Results of Sum-rate Vs SNR curve for hybrid Precoding mmW systems (a) comparison of different schemes, (b) varying the number of transmit antennas.

can give better throughput. Analyzing the proposed design under perfect channel, this approach has shown better per-formance compared to other hybrid precoding approaches.

\section{ACKNOWLEDGEMENT}

litera-ture discussed in the previous sections. The simulation is carried out in MATLAB simulator and in three different systems. MU-MIMO is considered in this analysis with BS antennas and MS antennas. Four mobile stations are considered and each having single-path trans-mission. The effective channel is assumed to be perfect with AoDs and AoAs to be uniformly distributed. The azi-muths and elevations are uniformly distributed in and respectively. Noise variance is assumed to be 1 and the SNR is . The achievable rates of the proposed hybrid MMSE analog/digital precod-ing technique is analyzed and compared with the single-user rate, analog beamforming and hybrid $\mathrm{ZF}$ precoding systems and results show that the proposed hybrid precod-ing scheme performs very close to single-user rate and ZF hybrid precoding and also achieves better gain over the analog beamforming approach. Fig. 1(a) demonstrates the achievable sum-rate of different precoding approaches. For massive MIMO mmWave systems, with a large number of antennas, the performance of the proposed scheme increases significantly. The simulation of the sum rate of the proposed hybrid precoding varying the BS antennas with fixed is shown in fig. 1(b).

\section{CONCLUSION}

In this paper, low-complexity hybrid precoding design is presented for mmWave multi-user communication with MMSE baseband precoding. For mmWave networks with perfect channel conditions, analog beamforming is not feasible due to low sum rate. Digital precoding system in-crease the performance but at the cost of high complexity. So, integrating both analog and digital processing in the BS

Authors are grateful to Research Management Center (RMC), International Islamic University Malaysia (IIUM) to support this research through research initiative grant scheme RIGS16-065-0229.

\section{REFERENCES}

1. Andrews, Jeffrey G., Stefano Buzzi, Wan Choi, Stephen V. Hanly, Angel Lozano, Anthony CK Soong, and Jianzhong Charlie Zhang. "What will 5G be?." IEEE Journal on Selected Areas in Communications 32, no. 6, 2014: 1065-1082.

2. Akdeniz, Mustafa Riza, Yuanpeng Liu, Mathew K Samimi, Shu Sun, Sundeep Rangan, Theodore S Rappaport, and Elza Erkip. "Millimeter wave channel modeling and cellular ca-pacity evaluation." IEEE journal on selected areas in com-munications 32 , no. 6 , 2014: 1164-1179.

3. Tsang, Y. Ming, Ada SY Poon, and Sateesh Addepalli. "Cod-ing the beams: Improving beamforming training in mmwave communication system." In Global Telecommunications Conference (GLOBECOM 2011), 2011 IEEE, pp. 1-6. IEEE, 2011.

4. Hosoya, Ken'ichi, Narayan Prasad, Kishore Ramachandran, Naoyuki Orihashi, Shuya Kishimoto, Sampath Rangarajan, and Kenichi Maruhashi "Multiple sector ID capture (MIDC): A novel beamforming technique for $60-\mathrm{GHz}$ band multi-Gbps WLAN/PAN systems." IEEE Transactions on Antennas and Propagation 63, no. 1, 2015: 81-96.

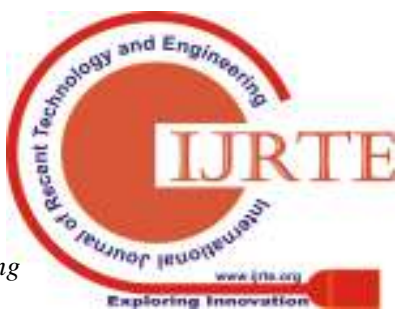


5. Hur, Sooyoung, Taejoon Kim, David J. Love, James V. Krogmeier, Timothy A. Thomas, and Amitava Ghosh. "Mil-limeter wave beamforming for wireless backhaul and access in small cell networks." IEEE Transactions on Communications 61, no. 10, 2013: 4391-4403.

6. Gholam, Fouad, Javier Vía, and Ignacio Santamaría. "Beam-forming design for simplified analog antenna combining ar-chitectures." IEEE Transactions on Vehicular Technology 60, no. 5, 2011.

7. Hampson, G. A., P. P. Roberts, M. Leach, A. J. Brown, T. Bateman, S. Neuhold, R. J. Beresford et al. "Microwave phased array digital beamforming system design challenges for SKA." In Microwave Conference (EuMC), 2015 European, pp. 710-713. IEEE, 2015.

8. Heath, Robert W., Nuria Gonzalez-Prelcic, Sundeep Rangan, Wonil Roh, and Akbar M. Sayeed. "An overview of signal processing techniques for millimeter wave MIMO systems." IEEE Journal of Selected Topics in Signal Processing 10, no. 3, 2016.

9. Kim, Chanhong, Taeyoung Kim, and Ji-Yun Seol. "Multi-beam transmission diversity with hybrid beamforming for MIMO-OFDM systems." In 2013 IEEE Globecom Work-shops (GC Wkshps), pp. 61-65. IEEE, 2013.

10. El Ayach, Omar, Robert W. Heath, Sridhar Rajagopal, and Zhouyue Pi. "Multimode precoding in millimeter wave MIMO transmitters with multiple antenna sub-arrays." In 2013 IEEE Global Communications Conference (GLOBECOM), pp. 3476-3480. IEEE, 2013.

11. Han, Shuangfeng, I. Chih-Lin, Zhikun Xu, and Corbett Row-ell. "Large-scale antenna systems with hybrid analog and dig-ital beamforming for millimeter wave 5G." IEEE Communi-cations Magazine 53, no. 1, 2015.

12. Huang, Xiaojing, Y. Jay Guo, and John D. Bunton. "A hybrid adaptive antenna array." IEEE Transactions on Wireless Communications 9, no. 5, 2010.

13. Alkhateeb, Ahmed, Omar El Ayach, Geert Leus, and Robert W. Heath. "Hybrid precoding for millimeter wave cellular systems with partial channel knowledge." In Information Theory and Applications Workshop (ITA), 2013.

14. Alkhateeb, Ahmed, Geert Leus, and Robert W. Heath. "Lim-ited feedback hybrid precoding for multi-user millimeter wave systems." IEEE Transactions on Wireless Communications 14, no. 11, 2015.

15. Liang, Le, Wei Xu, and Xiaodai Dong. "Low-complexity hybrid precoding in massive multiuser MIMO systems." IEEE Wireless Communications Letters 3, no. 6, 2014.

16. Chen, Chiao-En. "An iterative hybrid transceiver design algo-rithm for millimeter wave MIMO systems." IEEE Wireless Communications Letters 4, no. 3: 285-288, 2015.

17. El Ayach, Omar, Sridhar Rajagopal, Shadi Abu-Surra, Zhouyue Pi, and Robert W. Heath. "Spatially sparse precoding in millimeter wave MIMO systems." IEEE Transactions on Wireless Communications 13, no. 3: 1499-1513, 2014. 